\title{
Análisis de la mortalidad en ancianos en un servicio de Medicina Interna
}

\author{
S. CINZA SANJURJO, A. CABARCOS ORTIZ DE BARRÓN, E. NIETO POL ${ }^{1}$, \\ J. A. TORRE CARBALLADA \\ Servicio de Medicina Interna. Hospital Clínico de Santiago de Compostela. ${ }^{1}$ Servicio de \\ Atención Primaria Concepción Arenal. Santiago de Compostela. A Coruña
}

ANALISES OF THE MORTALITY IN AGED IN AN INTERNAL MEDICINE DEPARTMENT

\section{RESUMEN}

Objetivos: Establecer las características de los fallecimientos y causas de muerte en población anciana.

Material y métodos: Estudio descriptivo transversal, en el que se incluyeron pacientes mayores de 65 años ingresados en el año 2002 en un Servicio de Medicina Interna. Las variables analizadas fueron: edad, sexo, fecha de ingreso, fecha de alta, tiempo de estancia hospitalaria, patologías crónicas anteriormente diagnosticadas, motivo de ingreso hospitalario (síntoma guía por el que el paciente decide acudir al centro hospitalario), exitus intrahospitalario, diagnósticos clínicos al alta. En el análisis estadístico se emplearon índices estadísticos descriptivos de variables cualitativas y cuantitativas, Chi-cuadrado, Mann-Whitney-Wilcoxon y Kruskal-Wallis.

Resultados: Se recogieron datos correspondientes a 770 ingresos, de los que fallecieron 128, lo que representa un $16,6 \%$ de los ingresos. Se halló un predominio de sexo masculino $(53,1 \% ; 0,44-0,62 ; \mathrm{p}=0,48)$ y una edad media de 78,3 años $\pm 1,3$ años $(\mathrm{p}<0,001)$ (mediana de 79 años). La estancia media en el grupo de pacientes fallecidos fue de 13,3 días $\pm 1,7$ días $(\mathrm{p}<0,001)$ (mediana de 11,5 días). La muerte se produjo en las primeras 48 horas del ingreso en 5 pacientes $(3,9 \%)$.El principal motivo de ingreso en los pacientes fallecidos fue la disnea $(46,1 \%)$. Entre las patologías crónicas más prevalentes se encontraron: cardiopatía isquémico-hipertensiva $(18,8 \%)$ y EPOC $(14,8 \%)$. El diagnóstico más frecuente como causa de exitus fue la infección respiratoria $(43,8 \%)$.

Conclusiones: La prevalencia de patología crónica cardiovascular y pulmonar es elevada, además de ser las principales causas de mortalidad hospitalaria.

PALABRAS CLAVE: Muerte intrahospitalaria. Mayores de 65 años. Medicina Interna.
ABSTRACT

Backgroud. To establish the characteristics of the deceased and the death causes.

Methodology: Transversal study descriptive, with intake patients elder than 65 years old in an Internal Medicine Department. The variables analized were: age, sex, intake date, discharge date, days of hospital stay, chronic disease previous, admission cause, deceased, diagnoses. The statistical analysis was performed with measures of central tendency and of standard deviation, Chi-cuadrado, Mann-Whitney-Wilcoxon and Kruskal-Wallis.

Results: During the revised year, there are 770 patients intaked in Internal Medicine Department and 128 exitus (16.6\%). The global average death age was $78.3 \pm 1.3$ years: $53.1 \%(0.44-0.62 ; p=0.48)$ were men and $46.9 \%$ were women. The average death intake days was $13.3 \pm$ 1.7 days $(p<0.001), 3.9 \%$ died in less than forty-eight hours after hospitalization. The most frequent admission cause was: dyspnea (46.1\%). The most frequent chronic diseases were: ischemic and hypertensive heart disease (18.8\%) and chronic obstructive pulmonary disease. The most frequent death cause was respiratory tract infection (43.8\%).

Conclusions: The prevalence cardiac and pulmonary disease prevalence is high, these diseases are the of the most frequent causes hospital mortality.

KEY WORDS: Hospital mortality. Aged. Internal Medicine.

Cinza Sanjurjo S, Cabarcos Ortiz de Barrón A, Nieto Pol E, Torre Carballada JA. Análisis de la mortalidad en ancianos en un servicio de Medicina Interna. An Med Interna (Madrid) 2007; 24: 67-71.

\section{INTRODUCCIÓN}

La mortalidad hospitalaria es uno de los indicadores de calidad asistencial más frecuentemente empleado, ya que la cuantificación de las defunciones puede considerarse como una medida de la efectividad de la intervención hospitalaria aunque no se debe olvidar que está influenciada por otros fac- tores como, la patología atendida, la estructura poblacional, el régimen económico del centro y la accesibilidad al centro (1). Por otro lado, el análisis de la mortalidad puede utilizarse como un valioso instrumento para la planificación y gestión hospitalaria (2).

Las dificultades en la utilización de ratios de mortalidad global y ajustada, entorpece el poder realizar un análisis cuali-

Trabajo aceptado: 13 de octubre de 2006 
tativo de comparación entre hospitales, por este motivo en los años 80 la Health Care Financing Administration incorporó el análisis del porcentaje de mortalidad hospitalaria como comparación cualitativa de los hospitales americanos. Desde entonces han sido muchos los estudios publicados coincidiendo en la necesidad de ajustar la mortalidad a otros parámetros (3).

Habitualmente, para el análisis de mortalidad se emplean bases de datos administrativas a pesar de las limitaciones que presentan al no incorporar la gravedad del proceso (4). La más empleada es la del conjunto mínimo básico de datos (CMBD) y el estudio de mortalidad hospitalaria se realiza en función del diagnóstico principal según la clasificación Internacional de Enfermedades (CIE) (5), en otras circunstancias también se emplea el análisis de los Grupos Relacionados con el Diagnóstico (GRD) (6).

En nuestro centro hospitalario, se registra la mortalidad en base a los GRD y se refleja el diagnóstico principal, esta es la forma en que analizaremos en el presente estudio la mortalidad.

Según lo anteriormente expuesto, se propuso la realización del presente estudio la establecer las características clínicas de los ancianos fallecidos, así como la causa de muerte en un Servicio de Medicina Interna

\section{MATERIAL Y MÉTODOS}

Se realizó un estudio descriptivo transversal, en el que se incluyeron todos los pacientes mayores de 65 años ingresados a lo largo del año 2002 en el Servicio de Medicina Interna del Hospital Clínico Universitario de Santiago de Compostela, obteniéndose una población para estudio de 770 pacientes.

La recogida de datos se realizó mediante la revisión sistemática de los informes de alta/exitus y la posterior revisión de la historia clínica de los pacientes.

Las variables analizadas fueron: edad, sexo, fecha de ingreso, fecha de alta, tiempo de estancia hospitalaria, patologías crónicas anteriormente diagnosticadas, motivo de ingreso hospitalario (síntoma guía por el que el paciente decide acudir al centro hospitalario), exitus intrahospitalario, diagnósticos clínicos al alta.

Los motivos de consulta, antecedentes personales y diagnósticos al alta, se recogieron y codificaron según la CIE-9 (Clasificación Internacional de Enfermedades). Un mismo paciente puede presentar varios motivos de ingreso, tener más de una patología crónica conocida previamente, recibir más de un diagnóstico y necesitar más de un tratamiento.

Los datos recogidos se incluyeron en una base de datos de Excel 2003 diseñada para tal efecto, y el análisis posterior se realizó con el paquete estadístico G-STAT versión 2.0. En el análisis univariante se emplearon índices estadísticos descriptivos de variables cualitativas y cuantitativas. Para el análisis bivariante se empleó Chi-cuadrado, en el caso de variables cualitativas, Mann-Whitney-Wilcoxon y Kruskal-Wallis, en el caso de variables cuantitativas.

\section{RESULTADOS}

A lo largo del año 2002 se produjeron 770 ingresos en el Servicio de Medicina Interna correspondientes a pacientes mayores de 65 años, de los que fallecieron 128, lo que representa un $16,6 \%$ de los ingresos.
El análisis de los pacientes fallecidos muestra un predominio de sexo masculino $(53,1 \% ; 0,44-0,62 ; p=0,48)$ y una edad media de 78,3 años $\pm 1,3$ años $(\mathrm{p}<0,001)$ (mediana de 79 años). A pesar de que en la totalidad de pacientes ingresados predomina el sexo femenino $(51,1 \%)$, el predominio de varones en la población fallecida no es estadísticamente significativa ( $\mathrm{p}=0,29)$. Tampoco la edad presenta diferencias entre el grupo de pacientes fallecidos y los que no fallecen $(\mathrm{p}=0,57)$.

La estancia media en el grupo de pacientes fallecidos fue de 13,3 días $\pm 1,7$ días ( $\mathrm{p}<0,001$ ) (mediana de 11,5 días), sin que se hallaran diferencias estadísticamente significativas respecto a pacientes no fallecidos $(\mathrm{p}=0,91)$. El exitus se produjo en las primeras 48 horas del ingreso en 5 pacientes $(3,9 \%)$, sin hallarse diferencias estadísticamente significativas en cuanto sexo $(\mathrm{p}=0,57)$, edad $(\mathrm{p}=0,42)$ ni causa de muerte $(\mathrm{p}=0,12)$.

El $94,5 \%$ de los pacientes que fallecieron procedían de su domicilio, cifra similar a la presentada por los pacientes no fallecidos $(p=0,69)$. El recuento de fallecimientos por fecha de exitus muestra mayor número en los meses correspondientes a Primavera $(31,2 \%)$ frente al resto de épocas del año: $23,4 \%$ en Verano, $22,7 \%$ en Otoño y $22,7 \%$ en Invierno. A pesar de esta diferencia numérica, no se hallaron diferencias estadísticamente significativas entre fallecidos y no fallecidos $(\mathrm{p}=0,33)$. La distribución de los exitus por sexo y edad a lo largo de las estaciones anuales se muestran en las figuras $1 \mathrm{y}$ 2 , presentando diferencias estadísticamente significativas en el caso del sexo $(p=0,01)$, siendo en el caso de la edad no significativo $(\mathrm{p}=0,46)$.

Los principales motivos de ingreso en los pacientes que finalmente fallecieron fueron: disnea $(46,1 \%)$, síndrome general $(8,6 \%)$, fiebre $(8,6 \%)$, descenso del nivel de consciencia $(7,0 \%)$, náuseas y vómitos $(4,7 \%)$ y mareos $(4,7 \%)$ entre otros.

El 63,28\% de los pacientes fallecidos presentaban algún factor de riesgo cardiovascular y presentaban una media de 3,6 $\pm 0,3(\mathrm{p}<0,001)$ patologías crónicas conocidas previamente al ingreso. Entre las patologías previas más frecuentes se encontraban: cardiopatía isquémico-hipertensiva (18,8\%), EPOC $(14,8 \%)$, ninguna conocida $(12,5 \%)$, entre otras menos prevalentes; en la tabla I se presenta la prevalencia de las patologías crónicas presentadas por los pacientes que fallecieron.

Se realizaron una media de 3,4 40,3 diagnósticos por paciente, aunque solamente se analizó el diagnóstico principal como responsable del exitus. Los diagnósticos principales

\section{TABLA I}

PREVALENCIA DE PATOLOGÍAS CRÓNICAS EN PACIENTES FALLECIDOS

\begin{tabular}{lcc}
\hline & $\mathrm{n}$ & $\%$ \\
\cline { 2 - 3 } HTA & 62 & 48,4 \\
DM & 60 & 46,9 \\
Cp isquémico-hipertensiva & 24 & 18,7 \\
EPOC & 19 & 14,8 \\
Nada & 16 & 12,5 \\
Dislipemia & 13 & 10,1 \\
Enfermedad de Alzheimer & 8 & 6,3 \\
Infarto cerebral & 7 & 5,5 \\
Miocardiopatía estructural & 6 & 4,7 \\
Insuficiencia cardíaca & 5 & 3,9 \\
Fibrilación auricular & 5 & 3,9 \\
Otros & 38 & 29,7 \\
\hline
\end{tabular}




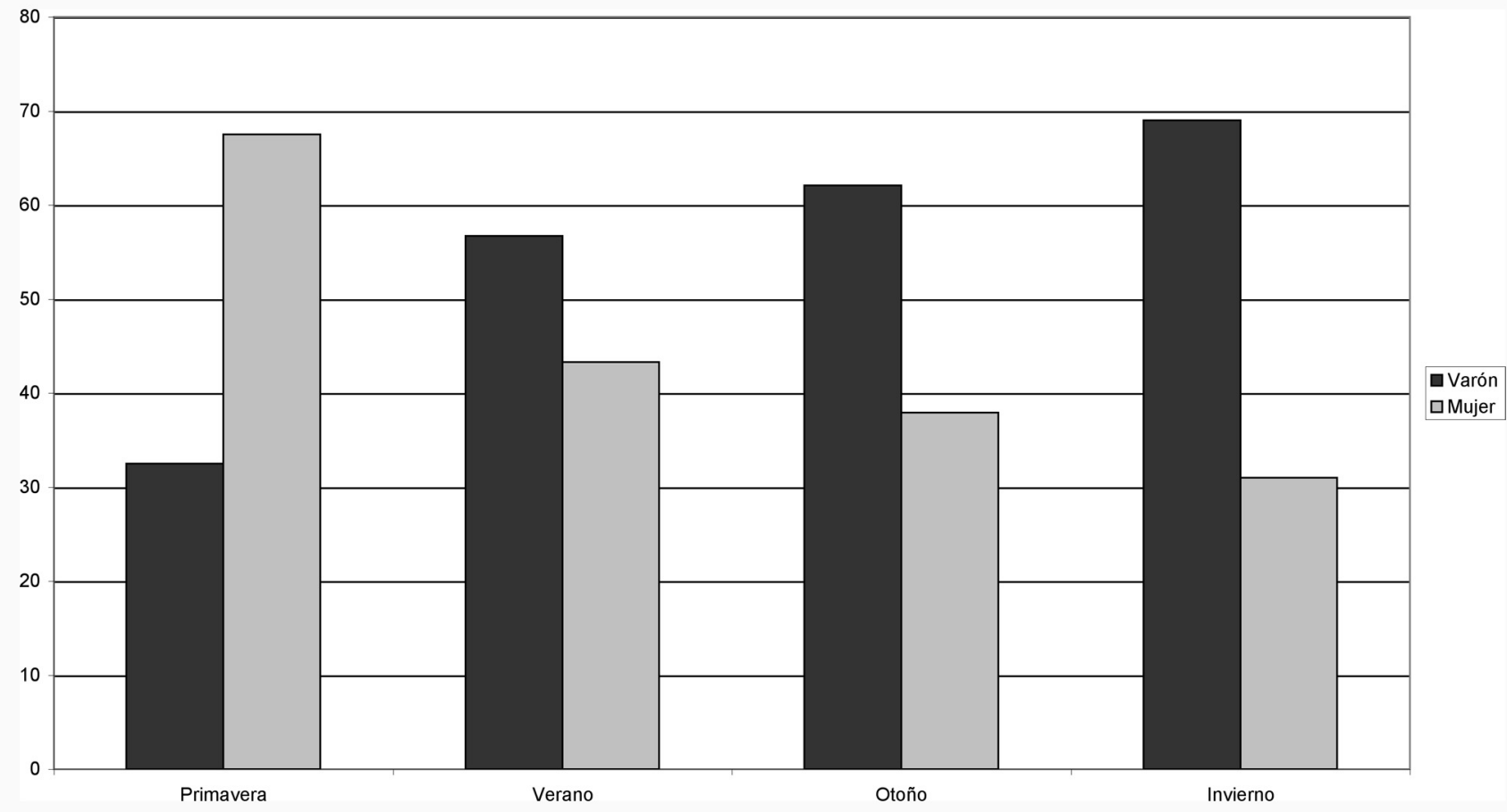

Fig. 1. Distribución de sexos por estaciones.

más frecuentes fueron: infección respiratoria $(43,8 \%)$, insuficiencia cardíaca $(13,3 \%)$, septicemia/bacteriemia $(4,7 \%)$, accidente cerebro vascular $(3,1 \%)$, infección urinaria $(3,1 \%)$, cáncer de pulmón $(3,1 \%)$, entre otros. En la tabla II se presentan con más detalle las causas de exitus.

\section{DISCUSIÓN}

El porcentaje de exitus producidos en el presente estudio $(11,6 \%)$ es superior al presentado en otros estudios y notablemente elevado en comparación al recomendado por el Instituto Nacional de Salud (4\%), lo que puede estar relacionado con las características demográficas (pacientes de edades avanzadas) y clínicas (pacientes pluripatológicos, con enfermedades de muchos años de evolución) de los pacientes estudiados (7).

El promedio de edad es elevado en el presente estudio, aunque tanto la distribución por sexo y edad es similar a la presentada por otros autores con población de estudio similar

\section{TABLA II}

\section{CAUSAS DE MUERTE}

Infecc. respiratoria baja

Neumonía

Insuficiencia cardiaca

Septicemia

Infección orina

Cáncer de pulmón

Accidente cerebro-vascular

Otros

\begin{tabular}{rr}
$\mathrm{n}$ & \multicolumn{1}{c}{$\%$} \\
\hline 29 & 22,6 \\
27 & 21,1 \\
17 & 12,3 \\
6 & 4,7 \\
4 & 3,1 \\
4 & 3,1 \\
4 & 3,1 \\
37 & 28,9 \\
\hline
\end{tabular}

a la nuestra $(1,3,8)$. Esta circunstancia se debe a que la población más joven que presenta patología de debut es manejada en unidades de cuidados intensivos o unidades más específicas (Cardiología, Neurología, Neumología, Digestivo, etc.), con lo que sólo se trasladan a Medicina Interna los pacientes pluripatológicos (generalmente de edad avanzada), más difíciles de manejar en estas unidades (9).

La neumonía o infecciones respiratorias son las patologías que han originado la mayoría de los ingresos y, a su vez, la mayoría de las defunciones, lo que se relaciona habitualmente con aspiración del contenido gástrico o de la papilla de alimentación en pacientes debilitados y postrados originando neumonías de más difícil tratamiento y peor pronóstico $(8,10$ 12). El resto de patologías causantes de los fallecimientos se producen por enfermedades muy prevalentes en nuestro medio, siendo similares a otras series $(1,3)$.

La mayoría de los pacientes presentaban numerosas patologías crónicas. La prevalencia de hipertensión o diabetes mellitus alcanza a casi la mitad de los pacientes ingresados. En el caso de patología cardiovascular o pulmonar alcanza un cuarto de la población ingresada. En el caso de otras patologías crónicas como infartos cerebrales o deterioro neurológico se presentaban en uno de cada 8 a 10 pacientes ingresados. Esta circunstancia ha sido descrita también por otros autores en un ámbito clínico similar al nuestro $(3,8,11,13)$.

Los Servicios de Medicina Interna se han convertido el lugar de ingreso de pacientes ancianos con múltiples patologías crónicas, los cuales con frecuencia fallecen durante el ingreso hospitalario debido a la descompensación de una o varias de estas patologías. Esta circunstancia se relaciona a un desplazamiento de la muerte del paciente desde su domicilio por parte de los familiares o de los centros geriátricos al cen- 


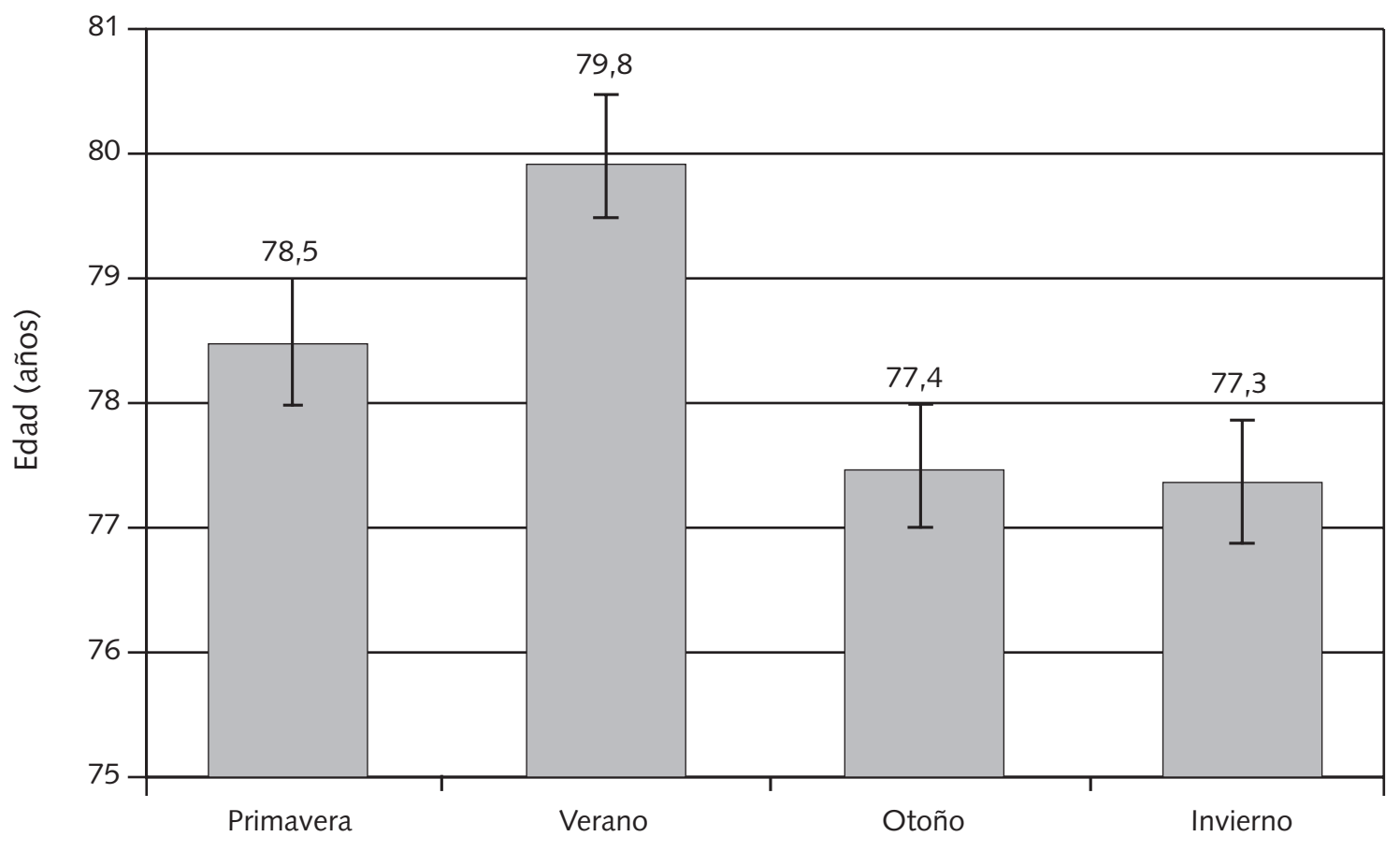

Estación anual

Fig. 2. Distribución de edades por estaciones.

tro hospitalario, hábito que también ha sido descrito por otros autores $(3,14)$.

La mortalidad en las primeras 48 horas se relaciona con factores extrahospitalarios, por lo que algunos autores prefieren realizar el estudio de pacientes que permanecen ingresados más de 48 horas. En nuestro estudio, se produjo el éxitus en este periodo en un 3,9\% de los pacientes fallecidos, porcentaje bajo que permite que se incluyan estos pacientes en el estudio (15).

Una de las limitaciones más importantes del presente trabajo es la no realización sistemática de necropsias a los pacientes fallecidos. Esta circunstancia se corresponde con el descenso que se está produciendo en los últimos años en la realización de esta práctica a nivel hospitalario (16). De todos modos, consideramos que no es una limitación importante del estudio ya que la correlación entre el diagnóstico clínico y el de la necropsia es elevado en grupos de pacientes como los que hemos incluido en el estudio, alcanzando cifras entre $85 \%$ y $94 \%$ (17). Los factores que influyen en el descenso de la realización del número de necropsias son múltiples, incluyendo tanto a la familia, los facultativos y la organización hospitalaria (7). Sin embargo, los beneficios científicos que aporta la necropsia son innegables, por lo que es necesario que se produzcan cambios en la actitud de todos los implicados en la necropsia, ya que es un procedimiento objetivo para detectar errores diagnósticos y es importante su utilidad como método de control de calidad18.

Otra posible limitación es que no se ha realizado distinción entre los exitus producidos por la patología crónica del paciente que motiva el ingreso y los exitus producidos por patologías diferentes a las propias del paciente o producidos por intervenciones diagnósticas o terapéuticas realizadas durante el ingreso. Esta situación es similar a la descrita previamente, ya que el porcentaje de exitus por procedimientos hospitalarios se encuentra en torno al $4 \%$ y los causados por otras patologías diferentes a las crónicas del propio paciente se encuentran entorno al 11\% (9). Aunque consideramos que es necesario efectuar investigaciones sobre este aspecto en los hospitales, ya que con los resultados que proporcionen podrían construirse nuevos índices de calidad en la atención hospitalaria.

Una de las críticas más importante consitiría en que en el informe realizado tras el exitus no figura qué pacientes son considerados terminales en el momento del ingreso, aunque bien es cierto que este dato sí figura posteriormente en la historia clínica desarrollada a lo largo del ingreso del paciente. El conocer qué pacientes son terminales en el momento del ingreso se relaciona con una actitud terapéutica encaminada a mejorar la calidad de la muerte (7).

Tras los datos expuestos previamente, se puede concluir que:

La prevalencia de patologías crónicas cardiovasculares es elevada en este grupo de pacientes, además son las principales responsables de la mortalidad hospitalaria en el mismo grupo.

La realización de revisiones similares a la actual ayuda a conocer la actividad realizada y a mejorar la calidad asistencial de los pacientes.

Es importante reforzar la realización de necropsias, que permitirá un mejor conocimiento diagnóstico de los exitus y es un importante parámetro de control de calidad. 


\section{Bibliografía}

1. García Ortega C, Almenara Barrios J, García Ortega JJ. Tasas específicas de mortalidad en el Hospital de Algeciras durante el periodo 19951996. Rev Esp Salud Pública 1997; 71: 305-15.

2. Segura Benedicto A. El análisis de la mortalidad hospitalaria como una medida de efectividad. Med Clin (Barc) 1988; 91: 139-41.

3. Del Río Fernández MC, Fernández Renedo A, de Frutos Arribas JF, Acebes Rey JM, Martín Escudero JC, Martínez Barrero F. Mortalidad en un Servicio de Medicina Interna de un Hospital de tercer nivel: veinte años de experiencia. An Med Interna (Madrid) 1998; 15: 197-201.

4. Ferrus Grau L. Estudio de mortalidad en un hospital comarcal. Todo Hospital 1996;129: 53-8.

5. Sánchez Bisono JR, Gómez-Rosich A, Amor Gea JF, García Sánchez MJ, Campy Domene LF, Peña Migallón-Sánchez P. Análisis de la mortalidad hospitalaria en un hospital comarcal. An Med Interna (Madrid) 1997; 14: 71-5.

6. García C, Almenara J, Mérida FJ, Serrano P. Tasas de letalidad por GRD en un hospital general básico. Rev Calidad Asistencial 2000; 15: 31-5.

7. Sanclemente C, Barcons M, Moleiro MA,Alonso F, Pañella D, Carrera R, Toribio R, Anglada A, Vilaró J. Mortalidad hospitalaria en un Servicio de Medicina Interna. An Med Interna (Madrid) 2004; 21: 317-21.

8. Viana A, Carballo F, Beato I, Domínguez E, Martínez C, De la Morena J. An analysis of 14.000 patients admitted to an internal medicine service during 1982-1988. An Med Interna (Madrid) 1990; 7: 459-62.

9. Vega J, Parodi M J, Puebla C. Relación entre el motivo de ingreso y la causa de muerte en pacientes fallecidos en un Servicio de Medicina Interna. Rev Med Chile 2004; 132: 707-17.
10. Sánchez J, Gómez A, Amor J, García M, Campoy L, Pena P. Analysis of hospital mortality at a regional hospital. An Med Interna (Madrid) 1997; 14: 71-5.

11. Rodríguez G, Fernández C, Delgado A, Carrasco M, Andradas E, De Juan $\mathrm{S}$ et al. Relationship between nosocomial infection and hospital mortality. Multicenter study. Med Clin (Barc) 1993; 100: 9-13.

12. Alonso J, Abinzano M, Martínez C, García M. Morbidity and mortality among the hospitalized aged. Identification of prognostic factors. An Med Interna (Madrid) 1995; 12: 420-4.

13. Matorral P, Daroca R, Alonso F, Ledesma F, Díaz-Caneja N, Gancedo Z. A morbidity study in general internal medicine service in a third level hospital. An Med Interna (Madrid) 1989; 6: 472-5.

14. Beguin C, Boland B, Van Mullem X, Van Mullem T, France F, Coche E et al. Fiftheen years of in a patient care in an academic division of general internal medicine: 1980-1994. Trends over time in patient's diagnoses, case-severity, case-complexity and mortality rate. Acta Clin Belg 1996; 51: 311-9.

15. Longo DR, Bohr D. Métodos cuantitativos en la gestión de la calidad. Una guía práctica. Barcelona: SG editores, 1994.

16. Ferguson RP, Burkhardt L, Hennawi G, Puthumana L. Consecutive autopsies on an internal medicine service. South Med J 2004; 97: 335-7.

17. De Escalante B, Oncins R, Sampedro J, Lacasa J, Figueras C, Najar M. Descriptive study of autopsies of internal medicine department at the hospital of Babastro and clinico-pathological correlation. An Med Interna 2000; 17: 460-4.

18. Abraham E. Autopsies in the UCI: we still need them. Crit Care Med 1999; 27: 235-6. 